\title{
Mobile Antenna Inetvu 1800+ Series and its Implementation in Practice
}

\author{
Marek Nízkya, Miloš Orgoña \\ ${ }^{a}$ Institute of Telecommunicatons, Department of Telecommunications, Slovak Universityof Technology, \\ Ilkovicova 3, Bratislava, Slovakia
}

\begin{abstract}
Although fiber-optic communication is used by a large number of telecommunication companies, satellite technology has still its important place among communication technologies. This article focuses on mobile antenna InetVu 1800+ Series. Besides detailed description of hardware and software components, functionality testing and troubleshooting of the whole system are included as the important parts of the topic.
\end{abstract}

Index Terms: Mobile antenna, antenna control unit (ACU), changing of azimuth and elevation on ACU, sensitivity of the system, management of the ports, GPS sensor. antenna isolation test.

(C) 2016 Published by MECS Publisher. Selection and/or peer review under responsibility of the Research Association of Modern Education and Computer Science

\section{Introduction}

Hardware part of the system was designed due to main request for using the antenna in variety terrain which includes windy and rainy weather conditions. The antenna should be ready for using by time less than 6 minutes, which depends on locality where the antenna is deployed. The Mobile Antenna InetVu 1800+ Series, which is shown in Figure 1, is fed from the black box (Figure 2), which is plugged directly into electricity network.

IDirect modem X5 is used for transmitting and receiving of the signal. Antenna Control Unit (ACU) is main device to control the whole system. Network part, which is important for user, is solved by a Mikrotik router.

Software part consists of Mikrotik router configuration, iDirect modem X5 configuration and ACU settings for what the program InetVu 7000 Series was designed. All parameters differ due to location where the antenna is used and satellite for the particular beam received by the antenna.

The main advantage of designed system is its application in different and inaccessible terrain. There are only two requirements:

* Corresponding author:

E-mail address orgon@ktl.elf.stuba.sk; marekmn@gmail.com 
1. access to electricity network,

2. direct visibility of the satellite.



Fig.1. Mobile Antenna 1800+ Series [1].

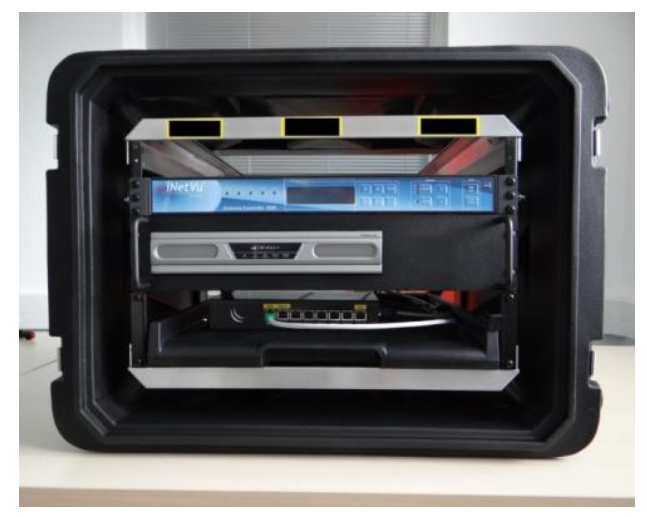

Fig.2. Devices: Mikrotik, iDirect X5 and Antenna Controller Unit.

\section{Helpful Hints}

System consists of the mobile Antenna with dish, which has diameter 1,8 $\mathrm{m}$. The antenna has 4 ports:

1. Port RX which is used for receiving of signal,

2. port TX which is used for transmitting of signal,

3. port for motor controlling,

4. port for powering of system.

The antenna has Block Up Converter designed by company NJRC (Figure 4), which has output 5 Watt and it is connected directly into iDirect modem X5. Antenna is able to work in three frequency bands: C-Band (4-8 $\mathrm{GHz})$, Ku-Band (11,2 - 14,5 GHz) and X-Band (8 - 12 GHz).

Color figures will be appearing only in online publication. All figures will be black and white graphs in print publication. 


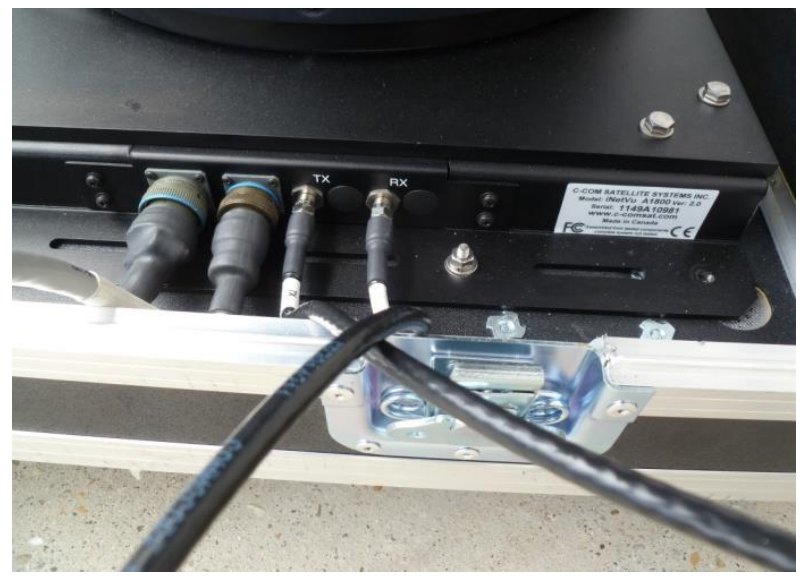

Fig.3. Antenna connectors

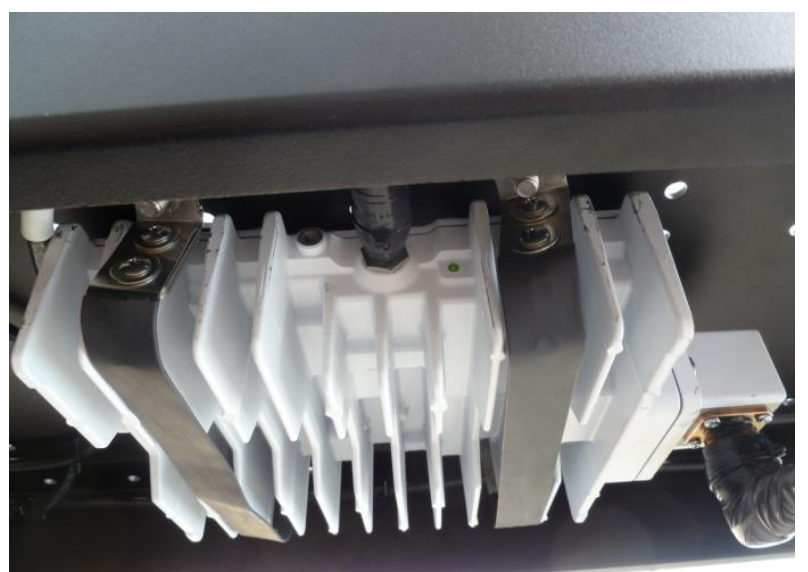

Fig.4. Block Up Converter NJRC.

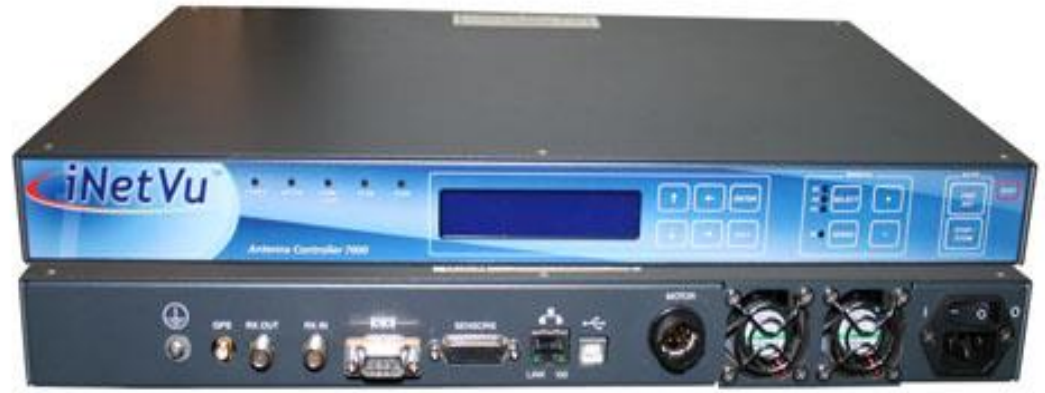

Fig.5. Antenna Control Unit device [2].

Antenna control unit shown on the Figure 5 is main device to control the whole antenna. First step, which is needed to synchronize ACU with iDirect modem X5 and Mikrotik router, is to configure software via 
iNetVu7000 Series (Figure 6). Configuration process consists of IP address changing from range of addresses dedicated for the customer. Besides IP address, mask and gateway need to be configured. Next step is choosing modem from the list and password adding to protect the ACU. Final step is to input frequency, symbol rate, offset and longitude of the satellite. After configuration is done, ACU is ready and the antenna can be stowed by button directly on the front panel.

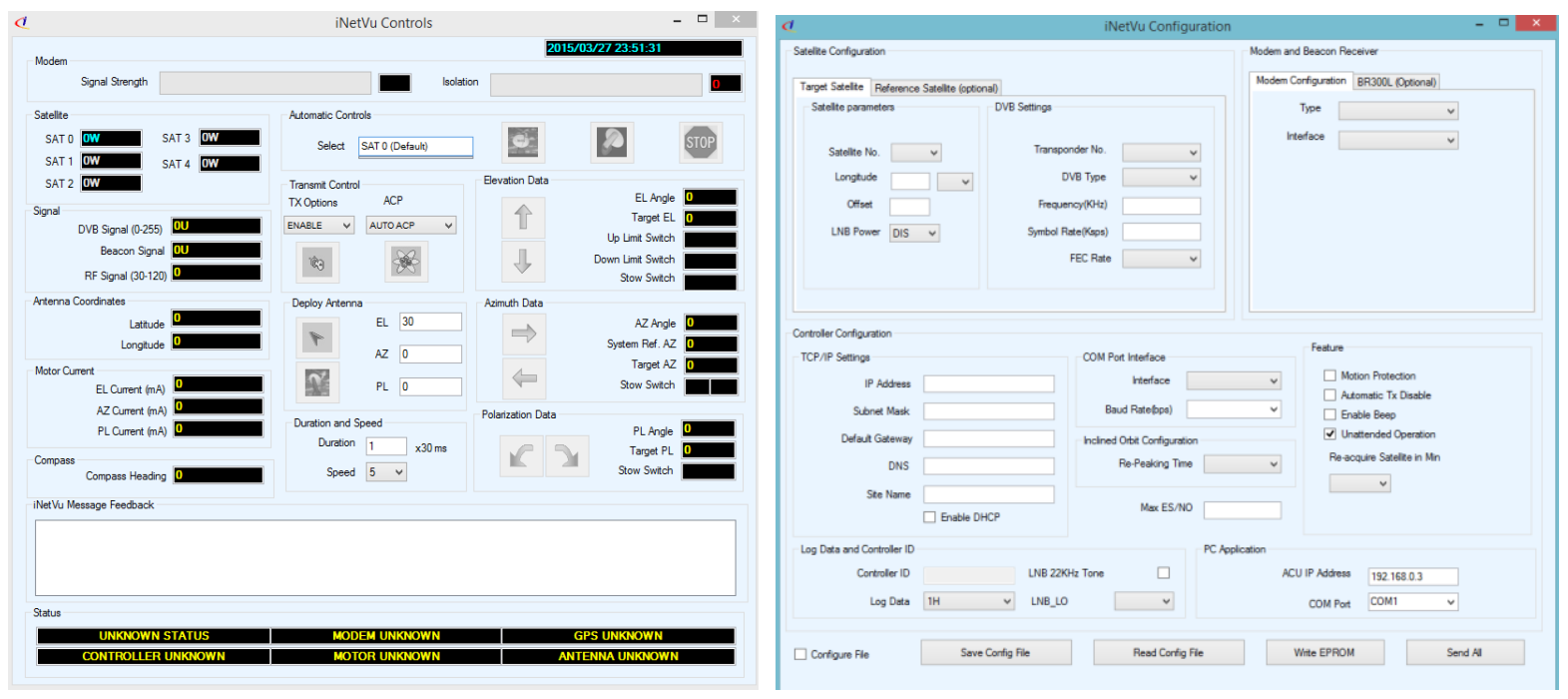

Fig.6. iNetVu 7000 Series - Web interface.

Antenna is searching several minutes until the satellite is found. After that, ACU is showing basic values of signal, values of azimuth and elevation and in case of $\mathrm{Ku}$-Band also value of polarization. Customer can reach better values of signal by slightly changing of azimuth and elevation on ACU.

Sensitivity of the system, how fast the antenna is stowing, powering of signal and other settings can be changed via Web interface (Figure 6). Moreover, ACU has ability to remember last running configuration which is very useful when antenna is deployed in the same location regularly. All information can be also found via display of ACU.

Figure 7 is showing one of the most widely used satellite modem - iDirect X5, which is easy to use and easy to configure. Moreover, as indicated above, it provides power to low-wattage power amplifiers. iDirect can be configured via computer with software iSite (Figure 8). It is necessary to configure the modem according to configuration on provider's side. In other words, some of the configured parameters have to be the same on both sides in order to maintain reliable connection. Configuration is done by uploading "Configuration file" into iDirect modem. This file includes definition for all necessary parameters such as data rates, the satellites, IP addresses etc. It is also necessary to change the login password, which must be the same as the password set on the device ACU. After recording configuration and the device restart, the modem is ready to use. The main advantage of this type of modem is its ability to adjust power according to measurement of the signal-to-noise ratio (SNR) meaning that in case SNR is too low modem automatically increases transmit power. Parameters SNR and power can be seen directly in the program iMonitor, which is used for monitoring on provider's side. The program records basic information about value of delays, Eb/No etc. According to gathered information provider is able to find the reason for an outage and troubleshoot issues on the link. If it is needed, customer can check the LED status on front panel of iDirect X5 modem as it is shown on Figure 7. Green light indicates no problem, orange light stands for problem which is present either on transmitting or receiving part of connection. 




Fig. 7. iDirect modem X5 [3].

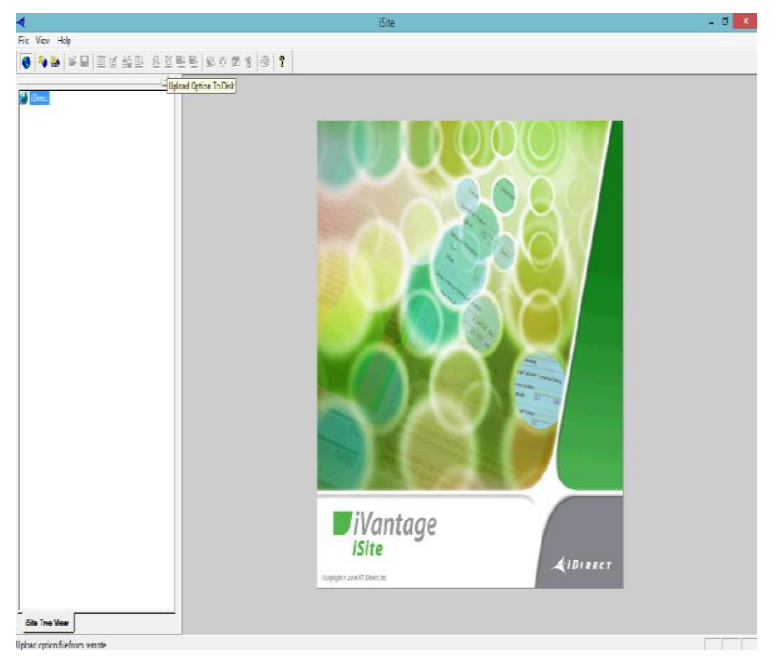

Fig.8. iSite menu

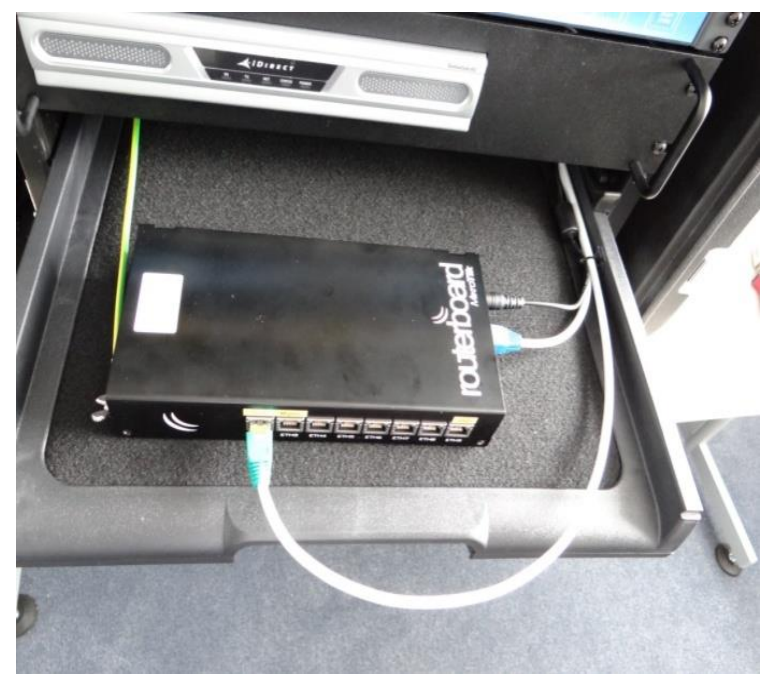

Fig.9. Mikrotik router. 
The last device which is used in system is Mikrotik router, which is shown on Figure 9. The device consists of Ethernet ports labeled Eth1 to Eth 9, serial interface and power interface that supplies electricity to the router. The configuration can be done in two ways. First option is by using software WinBox while there is a PC connected to the router via LAN cable. Second option is to plug the PC to the router using serial cable and terminal monitor program such as Putty.

In order to maintain simple management of the ports, it is recommended to configure the according to description below:

1. Eth1 is used to connect the satellite modem,

2. Eth2 is used to connect the device ACU,

3. Eth3 is used for network management,

4. Eth 9 is used to connect the user to the network.

\section{Description of Antenna Instalation}

Physical process of antenna installation mainly consists of antenna assembly testing. Firstly, it is necessary to connect the cables into the interface on the antenna (Figure 3), then the antenna arm is lifted by using the ACU, as it is showing in Figure 10.

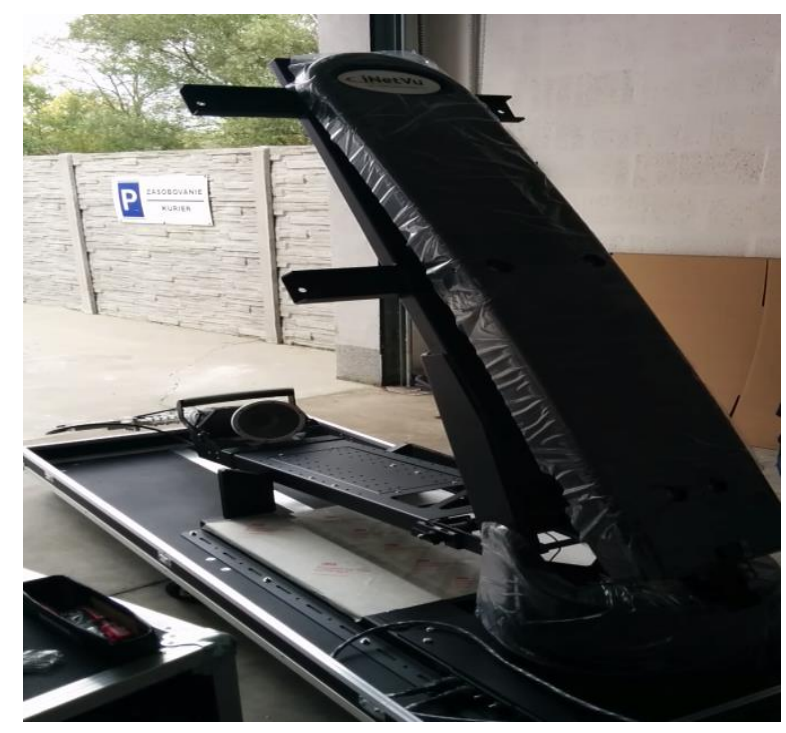

Fig.10. Arm of the Antenna. 


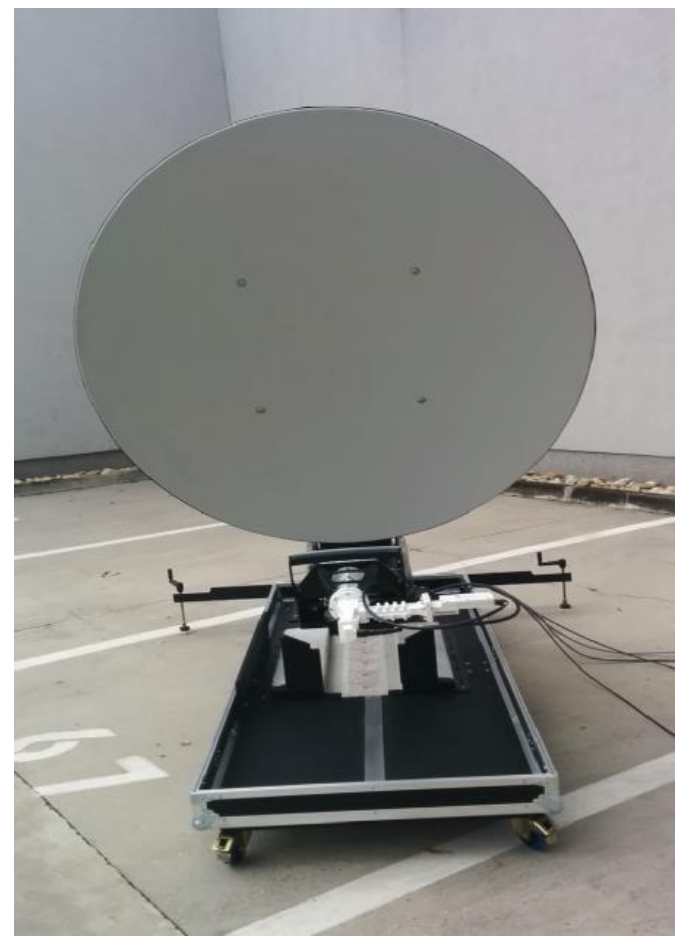

Fig.11. Dish of the antenna

After that antenna dish needs to be fixed by scrubs. It is necessary to install wider part of the dish into vertical direction due to antenna offset. In case wider part of the dish is installed into horizontal direction, antenna will not work.

The last step is to plug GPS sensor which is needed to locate antenna's position. Once the GPS coordinates are loaded the antenna is ready to test its functionality.

The antenna should be able to find the satellite up to several minutes after it has been stowed. Unfortunately, during our real testing we noticed that finding satellite can be difficult even if the antenna has a clear view up to the sky.

After several unsuccessful attempts, it was necessary to find a solution for this problem. The issue was solved radically - by installing new firmware to the ACU device. After that, the antenna was able to find the satellite, however it was not able to log into the network which brought another possible issue into the light. This issue was also solved by installing the new firmware on the satellite modem. After these steps the antenna was able to log into network, however another problems were found. Firstly, there were problems with cables length. When the antenna was tested in Slovakia, there was no degradation of signal but when the antenna was stowed in Africa, degradation by $2 \mathrm{~dB}$ of signal appeared. This problem was solved by using shorter, $15 \mathrm{~m}$ long cables.

The second problem which came up was low output power of Block Up Converter. Unfortunately, this issue is not so easy to solve as the power is provided to the converter from the modem. As every device, even modem has its limitation and the maximum power it is able to provide is 5 Watts.

Thirdly, there was problem with high elevation of the Antenna which was around 90 degrees because of geostationary satellite which is directly on the equator.

Last but not least, there was problem with bad placement of connectors on the antenna, which is also shown in Figure 3. 


\section{Mobile Antenna Testing}

After issues with the antenna was solved, we were testing system in different situation, which are usual for daily usage.

First test, which was done on the antenna was Antenna pointing and internet testing. During this test we were rotating the antenna by 90 degrees and we stowed the antenna in every position. Only one position was problematic - by rotating 180 degrees. It was because of functionality of system - antenna is not able to rotate by 360 degrees. In this position it took more than 8 minutes for antenna pointing.

Secondly the power issues were tested. We were trying to turn off electricity in different time - during antenna stowing, during pointing and at last when the antenna was pointed and locked into the network. In all of these different situations antenna was able to locate the satellite and bring the link status on.

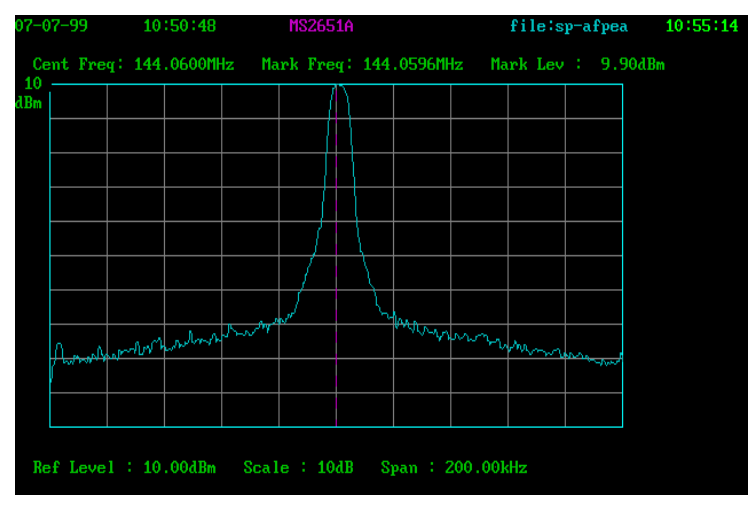

Fig.12. Clean carrier [4].

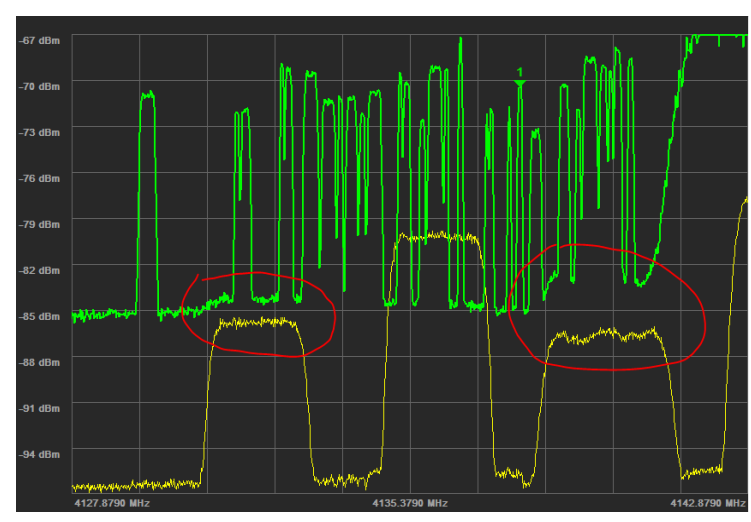

Fig.13. Basic interference.

Last part of testing included tests with satellite operator. There are two types of test: $1 \mathrm{~dB}$ compression and antenna isolation test. To perform $1 \mathrm{~dB}$ compression test, clean carrier (Figure 12) has to be turned on. During this test satellite operator is testing Block Up Converter saturation. Result of the $1 \mathrm{~dB}$ compression point test is a value of transmit power when the converter can no longer work according to linear function. With isolation test operator is trying to find potential problem with interference of other users. How interference looks alike can be found in Figure 13. Both of these tests were successful and our antenna was ready for using by customer. 


\section{Problem Solving During Antenna Deployment}

During antenna testing several rules are needed to be followed:

1. Antenna must has direct visibility on satellite. It is necessary to choose the most appropriate satellite. Footprints for every satellite can be found on the internet.

2. Grounding of whole system is very important in order maintain stable link without interruptions. How to ground every device can be found in Figure 14.

3. During antenna testing transmit power should be turned off to avoid health injuries caused by high signal transmitting because of Block Up Converter and also interfering of other users.

4. After antenna testing it is very important to tape very closely all connectors. Special volcanic adhesive tape is needed. It keeps the system waterproof during rainy weather conditions.



Fig.14. Devices grounding.

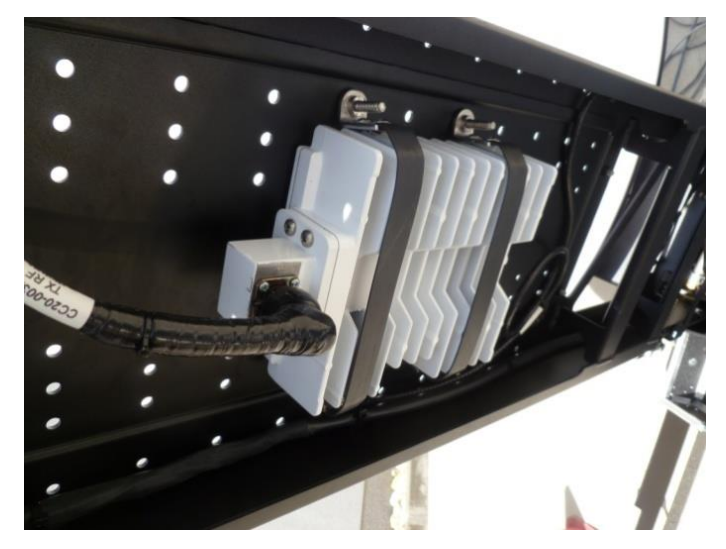

Fig.15. Taping of the connector

\section{Conclusions}

Subject of this paper was to test the implementation of the mobile antenna system iNetVu1800+ Series into the practice. Attention was paid to the proper selection of equipment, their configuration and consequently the elimination of errors that needed to be removed before starting the antenna. It was also needed to show which steps have to be done for success testing and the antenna using. 


\section{Acknowledgements}

This article was created with the support of the Ministry of Education, Science, Research and Sport of the Slovak Republic within the Research and Development Operational Programme for the project "University Science Park of STU Bratislava", ITMS 26240220084, co-funded by the European Regional Development Fund and his article is a part of research activities conducted at Slovak University of Technology Bratislava, Faculty of Electrical Engineering and Information Technology, Institute of Telecommunications, within the scope of the project KEGA No. 039STU-4/2013 "Utilization of Web-based Training and Learning Systems at the Development of New Educational Programs in the Area of Optical Transmission Media".

\section{References}

[1] http://www.c-comsat.com/inetvu-mobile-products/?Driveaway\#sub-type-Driveaways.

[2] http://www.groundcontrol.com/Mobile_1-8_Meter_C-Band.htm.

[3] http://www.groundcontrol.com/images/Evolution_X_X_Router_02.jpg.

[4] http://www.oh1sa.net/data/radio/yaesu/Yaesu_FT-736R_FT-757GX_FT-817ND_mods_and_repair_tips_ by_OH5IY/.

\section{Authors' Profiles}

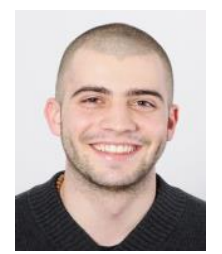

Marek Nízky was born in Stará L'ubovňa, Slovakia, in 1991. He received the B.E. degree in the Faculty of Electrical Engineering and Information Technology, Slovak Univesity of Technology in Bratislava in 2014. At present he is MsC. student in the Institute of Telecommunications FEI STU in Bratislava, Slovakia. His scientific research is focused on ZigBee networks.

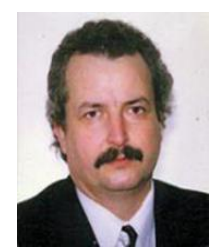

Miloš Orgoň was born in Piešt'any, Slovakia, in 1956. He received the Master degree and PhD. Degree in the Faculty of Electrical Engineering and Information Technology, Slovak Univesity of Technology in Bratislava in 1980 and 1988, respectively. Nowdays he works as an associate professor in the Institute of Telecommunications FEI STU in Bratislava, Slovakia. He has been engaged in research and development of telecommunication networks and services in liberalized environment for area of convergent technologies. At present he is currently engaged in research on the optimal design of networks and technological components, implementation of functions, services and applications and data security in the Research and Development Operational Programme for the project "University Science Park of STU Bratislava", ITMS 26240220084, co-funded by the European Regional Development Fund and his article is a part of research activities conducted at Slovak University of Technology Bratislava, Faculty of Electrical Engineering and Information Technology, Institute of Telecommunications, within the scope of the project KEGA No. 039STU-4/2013 "Utilization of Web-based Training and Learning Systems at the Development of New Educational Programs in the Area of Optical Transmission Media”. 